\title{
Magnetic Resonance Imaging and Image Analysis for Assessment of HPMC Matrix Tablets Structural Evolution in USP Apparatus 4
}

\author{
Piotr Kulinowski • Przemysław Dorożyński • Anna Młynarczyk • Władysław P. Węglarz
}

Received: 27 September 2010 / Accepted: 15 December 2010 / Published online: 23 December 2010

(C) The Author(s) 2010. This article is published with open access at Springerlink.com

\begin{abstract}
Purpose The purpose of the study was to present a methodology for the processing of Magnetic Resonance Imaging (MRI) data for the quantification of the dosage form matrix evolution during drug dissolution. The results of the study were verified by comparison with other approaches presented in literature.

Methods A commercially available, HPMC-based quetiapine fumarate tablet was studied with a 4.7T MR system. Imaging was performed inside an MRI probe-head coupled with a flowthrough cell for $12 \mathrm{~h}$ in circulating water. The images were segmented into three regions using threshold-based segmentation algorithms due to trimodal structure of the image intensity histograms.

Results Temporal evolution of dry glassy, swollen glassy and gel regions was monitored. The characteristic features were observed: initial high expansion rate of the swollen glassy and gel layers due to initial water uptake, dry glassy core disappearance and maximum area of swollen glassy region at $4 \mathrm{~h}$, and subsequent gel layer thickness increase at the expense of swollen glassy layer.

Conclusions The temporal evolution of an HPMC-based tablet by means of noninvasive MRI integrated with USP Apparatus 4
\end{abstract}

P. Kulinowski $(\bowtie) \cdot$ A. Młynarczyk $\cdot$ W. P. Węglarz

Department of Magnetic Resonance Imaging

Institute of Nuclear Physics Polish Academy of Sciences

ul. Radzikowskiego 152

3।-342 Kraków, Poland

e-mail: Piotr.Kulinowski@ifj.edu.pl

\section{P. Dorożyński}

Department of Pharmaceutical Technology and Biopharmaceutics

Pharmaceutical Faculty, Jagiellonian University

ul. Medyczna 9

30-688 Kraków, Poland was found to be consistent with both the theoretical model based on polymer disentanglement concentration and experimental VIS/ FTIR studies.

KEY WORDS flow-through cell · HPMC · image analysis . magnetic resonance imaging · USP Apparatus 4

$\begin{array}{ll}\text { ABBREVIATIONS } \\ \text { CR } & \text { controlled release } \\ \text { FOV } & \text { field of view } \\ \text { FTIR } & \text { Fourier transform infrared } \\ \text { FTIR-ATR } & \text { Fourier transform infrared-attenuated total reflection } \\ \text { HPMC } & \text { Hydroxypropylmethylcellulose } \\ \text { MR } & \text { magnetic resonance } \\ \text { MRI } & \text { magnetic resonance imaging } \\ \text { TE } & \text { echo time } \\ \text { TR } & \text { repetition time } \\ \text { USP } & \text { United States Pharmacopoeia } \\ \text { VIS } & \text { macro-photography }\end{array}$

\section{INTRODUCTION}

Hydrophilic polymer matrix systems are widely used for controlled release (CR) formulations (1). The distribution and mobility of water within the polymeric systems and their influence on properties of particular regions of the matrix during hydration are key factors for the identification of drug dissolution and transport mechanisms. A number of theoretical studies that attempt to develop models of these mechanisms are present in the literature and were recently reviewed by Grassi et al. (2) and Sieppman et al. (3). Most of the studies concerned in vitro drug release prediction. This approach seems to be unsatisfactory because the literature 
shows examples of formulations with similar dissolution profiles but with very different biological characteristics (4). Therefore, a parameterization (quantification) of physicochemical behavior of the dosage form, e.g. swelling, erosion and other matrix changes occurring during dissolution study, was proposed (5).

Up to now, only some of the theoretical studies concerned matrix structure and its evolution. For example, Borgquist et al. (6) simulated evolution of PEO matrix systems, Kiil et al. (7) calculated evolution of the three moving fronts of HPMC systems, while Ju et al. (8) presented a comprehensive mathematical model describing swelling/dissolution behavior and drug release from uncross-linked hydrophilic matrices (e.g. HPMG) in dynamic medium conditions.

The model by $\mathrm{Ju}$ et al. predicts dynamic existence of three different regions: dry core, characterized by unhydrated regime; swollen glassy layer with very strong polymeric chains entanglement; and gel layer with strong entanglement (8).

While the number of theoretical reports on matrix structure is limited, many experimental approaches have been presented in the literature. Several authors (9-13) have presented studies on identification of the boundary regions (moving fronts) in polymeric controlled release formulations (polymer-water-drug systems) using optical methods.

In particular, Colombo et al. (9) introduced two sharp fronts: the glass transition boundary and the erosion boundary. At the glass transition boundary, the glassy material is transformed into gel matrix, while at the erosion boundary, the matrix completely disappears due to dissolution or erosion. A third front, the drug diffusion boundary, where the drug dissolves and starts diffusing, was suggested to be placed between these two fronts.

Almost concurrently, Gao and Meury (10) described a slightly different model based on changes in the waterpolymer properties inside the HPMC matrix. Gao and Meury (10) identified the following fronts within the swelling HPMC matrix structure: true water penetration boundary, phase transition boundary and erosion boundary. At the erosion boundary (dissolution front), the matrix completely disappears by dissolution or erosion. At the phase transition boundary, the glassy material is transformed into a gel matrix and becomes transparent. The true penetration boundary reflects the extent of water penetration through the glassy material. The glassy polymer contains a large number of channels and holes of molecular dimension (pores in the carrier). Solvent penetration through these pores occurs prior to glassy-rubbery transition (10).

In 2004, Van der Weerd and Kazarian (11) applied the FTIR-ATR microscopy method with solution flow to study the evolution of the pure HPMC matrix as a function of time. Subsequently, in 2008 they reported (12) the results of combined optical (VIS) and FTIR studies that demonstrated three boundaries inside the HPMC matrix and showed that the time evolution of boundaries was supplemented with evolution of water and drug concentration, consistent with Gao and Meury's (10) results.

In addition to optical and FTIR-ATR methods, Magnetic Resonance Imaging (MRI) is also a useful technique for the study of tablet dissolution, since it allows the real-time, noninvasive recording of the alterations of 3D spatial distribution of water in polymeric matrices. Furthermore, MR images provide information on water density and mobility $(14,15)$. In comparison with other imaging methods, such as CT, image contrast in MRI can be adjusted by using different pulse sequences and altering their parameters. This is because MR image intensity depends on sample proton density, diffusion, proton relaxation times $\left(\mathrm{T}_{2}\right.$ and $\left.\mathrm{T}_{1}\right)$ and the selection of pulse sequence parameters, such as echo time (TE) and repetition time (TR). For example, using spin-echo-based pulse sequences, it is possible to image highly hydrated polymers (gel) and solutions in which $\mathrm{T}_{2}$ is longer than $\sim 10 \mathrm{~ms}$. Using the same pulse sequence, it is also possible to obtain images of water penetrating the polymer matrix before the glassyrubbery transition. However, significant loss in signal intensity occurs due to $\mathrm{T}_{2}$ relaxation times shorter than $\sim 10 \mathrm{~ms}$. Spin-echo-based MRI does not allow measurements of solutions in a "dry" glassy polymer matrix with $\mathrm{T}_{2}$ shorter than $100 \mu \mathrm{s}$, a very low level of water in polymer matrix can be visualized with MRI using other pulse sequences designed to solid state MR imaging $(14,16)$.

In polymer hydration, water molecules behave as both plasticizer and solvent. Hydration leads to the transition of the polymer matrix from the initial glassy state to the final solution state. This process is accompanied by matrix swelling and erosion as well as drug diffusion and release. NMR signal and thus the MR image intensity depend on the dynamic state of the water-polymer matrix system providing the mechanism for direct spatial visualization of regions with specific properties at the molecular level. In this way, MRI allows for the quantification of both the evolution of the region's sizes as well as the development of fronts (boundaries) between the regions within the HPMC matrix. It should be noted that the use of MRI in the visualization of the multiple regions in a hydrated polymer matrix requires the correct choice of parameters of the measuring method (TE, TR) in order to achieve measurable contrast between different regions (16).

In the 1990s, there were several attempts to use MRI for investigation of HPC/HPMC hydration (17-23) in nonpharmacopoeial conditions. However, according to our knowledge, there was only one report (published by Kojima et al. in 1998) (18) on observations of the additional interface layer between the dry core and the swollen gel layer, as well as evolution of all three layers. Recently, Chen 
et al. (23) showed interpretation of the $\mathrm{T}_{2}$ images of HPMC tablets in terms of dry core, swollen glassy and gel layers.

An important issue in completing MRI studies of dosage forms is to assure reasonable dissolution conditions. This could be accomplished by using one of the standard dissolution methods, i.e. usage of dedicated USP Apparatus 4 compatible probe-head (24-27). In our previous research, a dissolution Apparatus 4 integrated with an MRI system was used for imaging of non-compressed HPMC matrix systems during the dissolution process. This arrangement allowed assessment of the parameters related to swelling, diffusion and erosion fronts $(5,25,26,28)$. The concept of three moving fronts, introduced by Gao and Meury (10), for the first time was applied to the analysis of MRI data obtained inside the flow-through cell in 2005 (28). The interface region between dry polymer and gel was identified and marked as "hydrogel formation region" (26), yet there was no justification for this assertion.

On the contrary, in the MR micro-imaging study performed in the non-pharmacopoeial flow-through cell by Tajarobi et al. (29), the interface (i.e. swollen glassy) region was not identified. Only two fronts, erosion and swelling front, were assessed.

In recent years, pharmaceutical sciences have expanded their interest into new imaging techniques, including MRI (30). However, image processing and analysis are rarely discussed in the pharmaceutical literature. The main problem in the analysis of MR images of hydrating polymeric matrices is proper identification of regions characterized by different physico-chemical properties.

In particular, the aim of the study was

- to show a method of MR image processing providing quantitative information on evolution of the system during drug dissolution study under dynamic flow condition (USP Apparatus 4)

- to verify the results by comparison and discussion with the previous theoretical and experimental studies.

\section{MATERIALS AND METHODS}

\section{Materials}

Commercially available Controlled Release (CR) quetiapine fumarate $(400 \mathrm{mg})$ tablets were used. In the formulation of dosage form, the following excipients were used: hypromellose (HPMC), lactose monohydrate, microcrystalline cellulose, sodium citrate, and magnesium stearate.

\section{MR Imaging}

An MR research system equipped with MARAN DRX console (Oxford Instruments, UK) and a 4.7-T/310 mm superconducting magnet (Bruker, Germany) was used for imaging. The MRI study of the tablets was performed inside the homemade probe-head. The probe-head consisted of a flow-through cell (US Pharmacopoeia 4), RF resonator based on a saddle type RF coil and positioning holder (25,26). The experiments were performed in $1000 \mathrm{ml}$ circulating distilled water at a flow rate of $10 \mathrm{ml} / \mathrm{min}$, and the temperature was maintained at $37^{\circ} \mathrm{C}$. A modified spin-echo, flow-compensated pulse sequence was used to avoid artifacts from flowing dissolution medium. $\mathrm{T}_{1}$ and $\mathrm{T}_{2}$ weighted $\mathrm{MR}$ images were collected every $15 \mathrm{~min}$ over $12 \mathrm{hrs}$. The following parameters were applied: $\mathrm{FOV}=35 \times 35 \mathrm{~mm}, \mathrm{TE}=19 \mathrm{~ms}, \mathrm{TR}=625 \mathrm{~ms}$, sampling rate $20 \mu \mathrm{s}$, read gradient strength $33.7 \mathrm{mT} / \mathrm{m}$, slice thickness $1 \mathrm{~mm}$, number of averages 4, matrix size $256 \times 256$. Parameters of the sequence were chosen for imaging of hydrophilic HPMC matrices with incorporated soluble active pharmaceutical ingredient. Echo time (TE) was set to minimum available value, i.e. $19 \mathrm{~ms}$, to maximize the contribution from the less hydrated parts of the matrix. Repetition time (TR) was set short enough $(625 \mathrm{~ms})$ to partially suppress bulk fluid (due to its long $T_{1}$ relaxation time), and in consequence to obtain reasonable gel-bulk fluid contrast.

\section{Image Analysis}

MR signals were acquired during 12 hours with the same optimized acquisition parameters. After the reconstruction, MR images were saved in Analyze format preserving original image intensity, i.e. no scaling or normalization was performed. This allowed the entire dataset of images to be processed together using the same image processing procedures and parameters.

The images were analyzed using public domain software Fiji (http://pacific.mpi-cbg.de/wiki/index.php/Fiji), distribution of ImageJ v.1.44 (NIH, http://rsb.info.nih.gov/ij/).

Modality of the image intensity histogram of the dosage form region is self-evident (see Figs. $1 \mathrm{~B}$ and 2); for this reason, histogram-based thresholding was chosen as the method of image segmentation $(31,32)$. However, it was discovered that proper segmentation was impossible when bulk fluid was included in the image. In order to overcome this difficulty, the textural segmentation with some manual corrections was applied to separate and remove bulk fluid. Textural segmentation was performed with the Trainable Segmentation Plugin (33). The Plugin applies several filters (including a median with radius of 1, 2, 4 and 8 pixels, Sobel and Hessian) to the image; the resulting set of images is called "feature stack." The user selects samples (groups of pixels) and assigns them to a class - in our case, the classes were bulk fluid, dry glassy, swollen glassy and gel. Then the classifier, Fast Random Forest algorithm, is applied to the 




Fig. I MR image of HPMC-based controlled release quetiapine fumarate tablet at 2:45 h (A), image intensity histogram (B), binary images (CI: - dry glassy core; C2: swollen glassy matrix; C3: gel).

original image and feature stack. This segmentation approach is sensitive not only for intensity of particular pixels but also patterns of local intensity changes (texture) in the pixel's vicinity. This segmentation approach was applied to each image in the stack separately. Segmented binary images of bulk fluid after some minor manual correction were used as masks for removing bulk fluid from original images.

After removal of bulk fluid, a gaussian blur filter (with standard deviation of the distribution, $\sigma=3$ pixels) was applied to reduce small local intensity changes, preserving the image edges better than a similarly sized mean filter. Next, the threshold levels were assessed: the region of interest (ROI) containing two regions were selected manually-for example: dry glassy core and swollen glassy regions-and one of the global segmentation methods/algorithms was used. The procedure was performed for several images in the stack where the adjacent regions had reasonable areas and mean threshold level was calculated.

Dry glassy-swollen glassy modes threshold was estimated with Iterative Self-Organizing Data Analysis Techniques (ISOdata) algorithm. The threshold was set at 0.16 level
$(0.16 \pm 0.003$ as assessed for nine images from the stack). While the swollen glassy mode is relatively narrow, the gel mode is usually broad and consequently has lower amplitude; in this case, a triangle method was used. The threshold was set to 0.43 $(0.43 \pm 0.036$ as assessed for fourteen images from the stack).

All images in the stack were thresholded using the same two threshold levels - one separating glassy dry core and swollen glassy part of the matrix, the second separating swollen glassy and gel. The result was three series of binary images reflecting the evolution of segmented regions, i.e. dry glassy core, swollen glassy and gel regions. Finally, the areas of the regions were calculated. Consequently, the evolution profiles of the areas of the three selected regions were obtained. Two additional profiles were calculated: the total swelling profile, which reflects changes of the total crosssection area of the system, and the glassy core profile, which reflects changes of the total area of polymer in glassy state.

Fig. 1 shows an example of image segmentation for image recorded at 2:45 h. It was selected as an illustrative example because at this time point all three regions coexist and have comparable sizes.

Fig. 1A1 represents MR image in grayscale. Fig. 1A2 shows region of interest (ROI) containing hydrated matrix
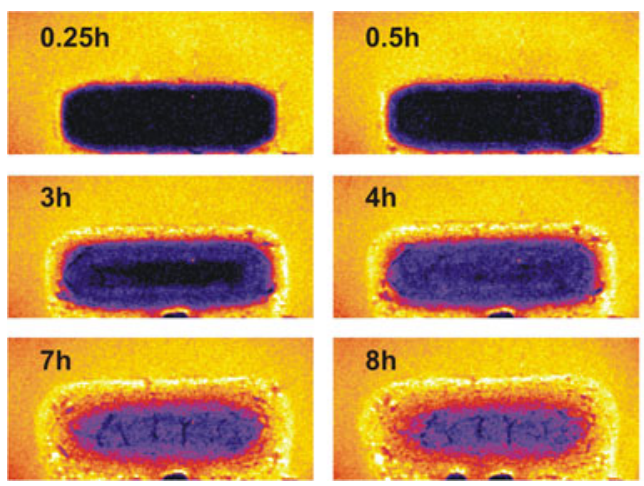
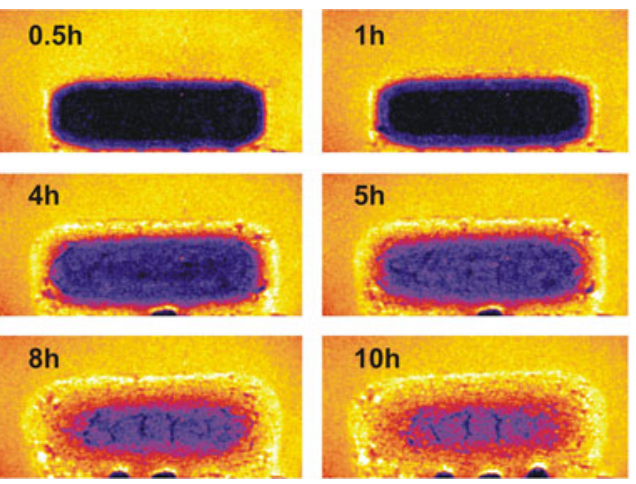
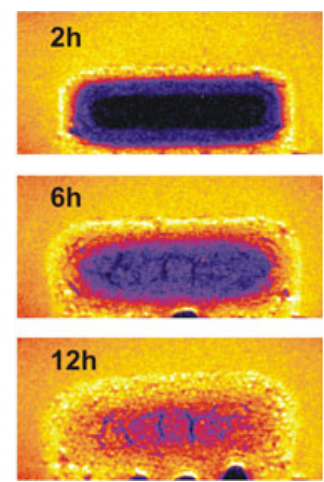

Fig. 2 An example of MR images of the quetiapine fumarate tablet during the hydration inside USP Apparatus 4 (TR/TE $=19 / 625$ ms). 
selected from the original image with convenient Lookup Table (LUT) applied. LUT can be an arbitrary color set. Application of LUT transformed grayscale image into a color image for presentation purposes in order to better visualize matrix structure (as in Fig. 3). It does not change actual values of pixel intensities - image analysis as described earlier was performed on original images. Fig. $1 \mathrm{~B}$ presents histogram of the image in Fig. 1A. The histogram consists of three distinct modes with appropriate assignment of physical properties (see "Discussion"). According to selected thresholds (0.16 and $0.43)$, original image was segmented yielding three binary images (Fig. 1G) of dry glassy (C1), swollen glassy (C2) and gel (C3) regions.

\section{RESULTS}

A set of MR images of the swelling tablet was collected during $12 \mathrm{~h}$ of hydration inside the flow-through cell. In the Fig. 2 examples of the acquired images are presented, an interface region (swollen glassy region) between dry glassy inner part of the table and the outer gel layer is clearly visible.

Another view of the changes inside the swelling HPMC matrix during hydration inside the flow-through cell is shown in Fig. 3.

Fig. 3 shows the histogram modes appearing and decaying during the dosage form hydration. We hypothesize that the modes shown in Fig. 3 reflect physico-chemical properties of the system as proposed by Ju et al. (8), i.e., dry glassy, swollen glassy and gel regions.

It can be observed that the swollen glassy mode is broader than dry glassy core, and the gel mode is substantially broader than the swollen glassy core (approximately 4 times broader). While homogeneity of a particular region is directly related to the mode width, it implies that the more hydrated the polymeric matrix, the more inhomogeneous its structure becomes in terms of solvent (water) concentration and

Fig. 3 Evolution of image intensity histogram (0: 15-12:00 h) of the quetiapine fumarate tablet during the hydration inside USP Apparatus 4 with temporal resolution of $15 \mathrm{~min}$. mobility, as well as differences in magnetic susceptibility (solvent/gel-air).

The evolution profiles of the regions are shown in Fig. 4.

At the beginning, total area of tablet cross-section was equal to the area of dry glassy core. Initially, the area of dry glassy core decreased with the rate of $0.976 \mathrm{~mm}^{2} / \mathrm{min}\left(R^{2}=0.9429\right)$ during the first $30 \mathrm{~min}$. Subsequently, the linear decrease of the dry glassy core with the rate of $0.28 \mathrm{~mm}^{2} / \mathrm{min}\left(R^{2}=\right.$ 0.9932 ) was observed. At 4 h, the dry glassy core disappeared completely.

The initial swollen glassy area growth rate was $0.99 \mathrm{~mm}^{2} / \mathrm{min}\left(R^{2}=0.9129\right)$. This decreased to $0.25 \mathrm{~mm}^{2} / \mathrm{min}\left(R^{2}=0.9899\right)$ at $30 \mathrm{~min}$, and the maximum of the swollen glassy area was reached at $4 \mathrm{~h}$. Between 4.25 and $10.25 \mathrm{~h}$, the linear decrease of swollen glassy area with rate $0.16 \mathrm{~mm}^{2} / \mathrm{min}\left(R^{2}=0.9983\right)$ was observed.

The increase of the gel area covering the tablet surface was observed over $12 \mathrm{~h}$. After the short period of initial gel formation (with rate of $0.98 \mathrm{~mm}^{2} / \mathrm{min}\left(R^{2}=0.9789\right)$ during the first $30 \mathrm{~min})$, the linear growth of the gel area with rate of $0.21 \mathrm{~mm}^{2} / \mathrm{min}\left(R^{2}=0.9966\right)$ was sustained for the period of $0.5 \mathrm{~h}$ to $10.25 \mathrm{~h}$.

During the first $30 \mathrm{~min}$, the total glassy (dry glassy+ swollen glassy) area remained approximately constant, while all other regions changed rapidly. Then, only a slight decrease with a rate of $0.07 \mathrm{~mm}^{2} / \mathrm{min}\left(R^{2}=0.9725\right)$ was observed up to $4 \mathrm{~h}$ after the beginning of the study.

The summary of the behavior of profiles is listed below:

- Initial high expansion rate (steeper slope) of the swollen glassy and gel layers within the first $30 \mathrm{~min}$ was observed due to the initial water uptake.

- Almost instantaneous rate change at 30 min resulted in a slower expansion of the swollen glassy and gel layers, the moment when a stable gel layer is formed.

- Dry glassy core disappeared at approximately $4 \mathrm{~h}$.

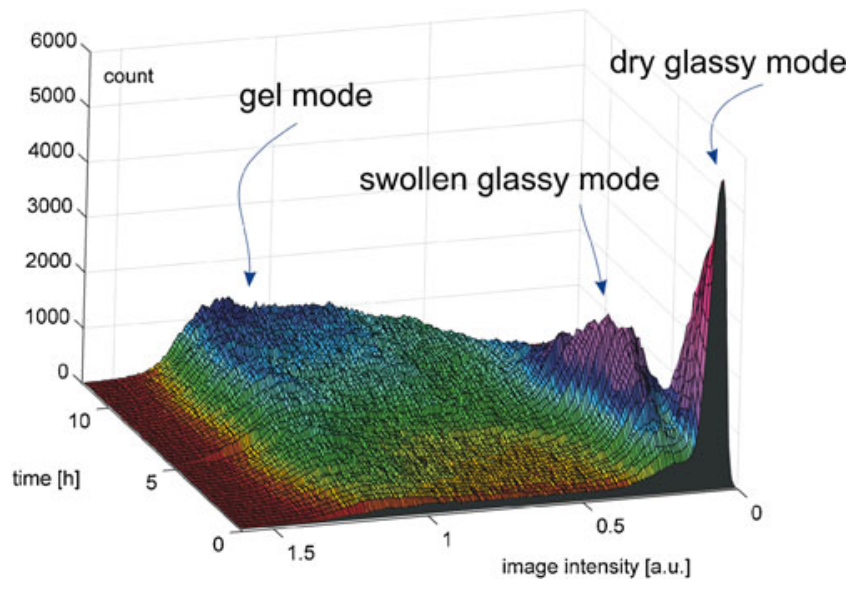


Fig. 4 Time evolution of the dry glassy core, swollen glassy layer, gel area and total area of the cross section of the HPMC based, CR quetiapine fumarate tablet as measured in the central saggital slice.

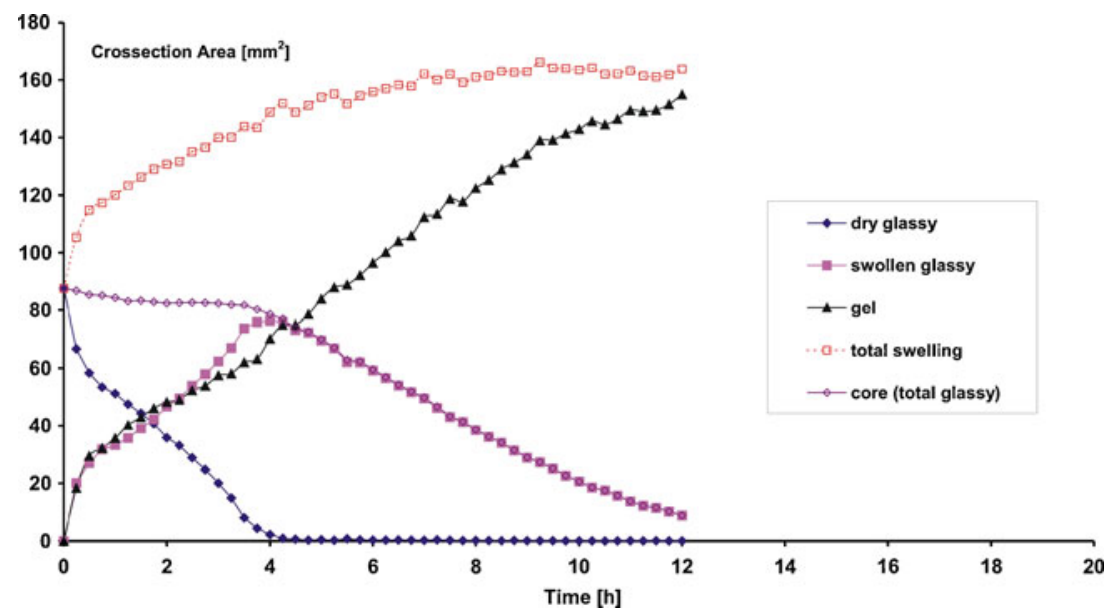

- Swollen glassy layer reached maximum at $4 \mathrm{~h}$, exactly at the time when the dry glassy layer disappeared.

- Starting from $4 \mathrm{~h}$, the gel layer thickness increased at the expense of the glassy swollen layer only.

\section{DISCUSSION}

The results described above, obtained using USP Apparatus 4 , agree with both the theoretical study presented by Ju et al. (8) and the combined VIS/FTIR study presented by Kazarian et al. (12). All three studies concern dynamic dissolution testing in either solvent flow or agitation states.

In their theoretical study, Ju et al. (8) calculated the time evolution of particular regions of the matrix for a dosage form (500 mg) comprised of 35\% HPMC (250 kDa and $10 \mathrm{kDa}$ ), 2.5\% adinazolam mesylate (water soluble drug), $62 \%$ lactose (binder) and $0.5 \%$ magnesium stearate (binder). The normalized changes in the thickness of the regions vs. time were presented. The model predicted the overall size of the matrix (total swelling of the system) for HPMC $250 \mathrm{kDa}$ would grow monotonically for the $20 \mathrm{~h}$. This slow dissolution rate of the polymeric matrix would result in the domination of solvent uptake over polymer dissolution. In addition, the model predicted that initially the swollen glassy layer and gel layer thickness would increase in an almost linear manner. At approximately $1 \mathrm{~h}$, the expansion rate of both fractions (swollen glassy layer and gel layer) would decrease, and at $5 \mathrm{~h}$, the thickness of the swollen glassy layer would reach its maximum extent, exactly when the dry glassy core disappeared. After this, the model predicted that the thickness of the swollen glassy layer would decrease until it finally disappeared at $16 \mathrm{~h}$.

Our results agree with the model developed by $\mathrm{Ju}$ et al. (8), as the behavior of the profiles presented in Fig. 4 demonstrate. This agreement between the model and our results is particularly evident for the HPMC $250 \mathrm{kDa}$ formulation. While both studies, theoretical and experimental, differ in details, constitutive similarities between the studies in terms of subjects and conditions include the following:

- HPMC polymeric matrix,

- dissolution medium (distilled water) in agitated/dynamic state,

- similar size of the dosage form,

- active substance soluble in water.

MRI parameters such as echo and repetition times (TE, TR) were selected to obtain proper contrast within the outer layer. The aim was to observe the gel layer, i.e. strong entanglement regime region, not the diffusion layer, characterized by a weak entanglement, to be in agreement with assumptions made by Ju et al. (8). Taking into account MRI experimental conditions in our study $(\mathrm{TE}=19 \mathrm{~ms}, \mathrm{TR}=$ $625 \mathrm{~ms}$ ), we may expect detection of signal from regions of the hydrating tablet with $\mathrm{T}_{2}$ above $\sim 8 \mathrm{~ms}$. This estimation is based on the assumption that at least $10 \%$ of the initial echo signal, decreased due to $\mathrm{T}_{2}$ decay, is detected. A spin-echo sequence with these parameters does not allow detection of very low hydrated regions, which are classified as part of the dry glassy core, leading to some overestimation of its size using MRI. Such a slight overestimation of the dry core area during hydration (Fig. 4), when compared to Ju's results (8), may be observed. This discrepancy could be minimized using shorter echo time. However, technical limitations, such as gradient strength, of our MRI system did not allow the use of shorter echo times. In spite of this technical limitation, the correlation between our experimental results (Fig. 4) and the theoretical predictions made by Ju et al. (8) is evident.

After dry core disappearance at about $5 \mathrm{~h}$ (Fig. 2), the voids/cracks appear in the swollen glassy part of the matrix. We hypothesize that they arise due to internal tensions in compressed polymer/drug/fillers matrix being released during hydration of the outer regions. When studying non-compressed matrices, maximum swelling occurred in 
the longitudinal dimension, and such voids were not observed (5). In the current study, the voids/cracks appear in the planes perpendicular to the longitudinal axis of the system (presumably perpendicularly to the direction of maximum internal tensions).

Kazarian et al. (12) applied combined VIS (visible photography) and FTIR with medium flow inside a nonpharmacopeial flow-through cell containing a tablet composed of HPMC (K4MRG) and BPP (buflomedil pyridoxal phosphate) $(60 \%)$. The three observed optical fronts were in agreement with the observations made by Gao and Meury (10). The true penetration front was located at low water concentration scale $(<<0.3 w / w)$ and was attributed to water penetrating through the channels inside the porous tablet with no or partial gelling of HPMC. In terminology introduced by Ju et al. (8), it encircles the dry glassy core. The second front, total gellification front, at approximately $0.6 w / w$ water content according to the FTIR results, separates the swollen glassy and gel regions. The erosion front appears at very high water concentrations and separates gel and solvent. The data, as presented by Kazarian et al. (12), transformed from front evolution (Fig. 5a) to the system evolution by means of layer thickness, are sketched in Fig. 5b.

Our results as presented in Fig. 4 concur with the profiles obtained by Kazarian (12) (Fig. 5b) with the dry glassy-gel interface evolution profile from Fig. 5b corresponding to the swollen glassy region evolution contained in Fig. 4.

However, the conditions of the studies by Kazarian et al. (12) - the sample restricted between plates or clamped between ATR crystal and transparent polymer windowand size of the matrix (small tablets of $3 \mathrm{~mm}$ diameter) did not fully reflect pharmacopoeial dissolution studies. In spite of this difference, the FTIR technique still gives unambiguous information concerning water and drug concentrations in particular fronts.

The correct identification of particular areas occurring in controlled release matrix systems during wetting allows us to develop an understanding of the mechanism of drug dissolution. It is particularly important when dealing with the original and generic controlled release formulations. This was emphasized in the summary report of the workshop entitled "Challenges and Opportunities in Establishing Scientific and Regulatory Standards for Assuring Therapeutic Equivalence of Modified Release Products" (34). At this workshop, it was concluded that improved prediction of the in viwo performance of modified release products is needed to understand the mechanism underlying the drug release from the dosage form. In the case of generic controlled release dosage forms, modified release mechanisms could not be the same as in the branded product; hence, it is unlikely that one dissolution method might be appropriate or predictive for both formulations. From this point of view, in vitro dissolution profile is only one of many elements describing in vivo properties. Structural evolution of the system contains additional information, which reflects mechanisms behind the drug dissolution $(4,5)$.

\section{CONCLUSIONS}

The presented paper describes an image analysis procedure applied to MR images of HPMC-based matrix tablet structure during hydration. The results show the first experimental observation of temporal evolution of HPMCbased matrix systems using MRI techniques integrated with USP Apparatus 4 consistent with the theoretical model presented by Ju et al. (8). Qualitative agreement between a)

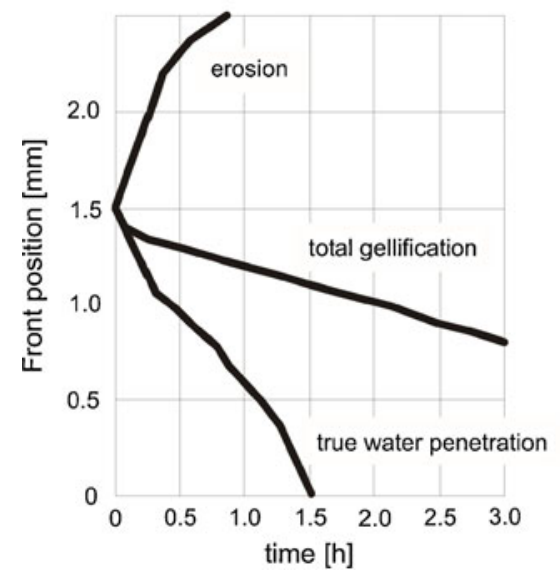

b)

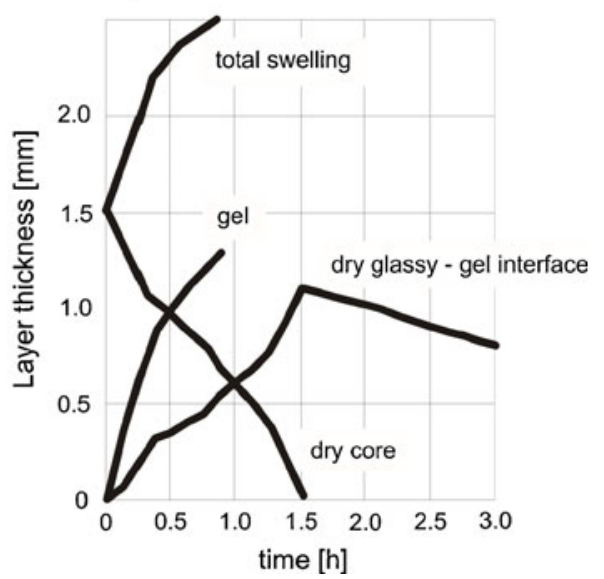

Fig. 5 A schematic of the HPMC matrix evolution according to VIS data according to data presented by Kazarian et al. ( I l ). Data were transformed from the front evolution (a) to the system evolution using layer thickness (b). 
experimentally obtained and simulated time evolution of the regions with different physicochemical properties, i.e. dry glassy, swollen glassy and gel regions, is shown. The results are also compared with VIS/FTIR experimental study (by Kazarian et al.) (12). The three complementary approaches, pharmacopoeial MRI in flow-through cell, theoretical modeling of HPMC, and VIS/FTIR, were found to provide consistent results in terms of temporal evolution of the matrix.

\section{ACKNOWLEDGMENTS}

The work was supported by the Polish Ministry of Science and Higher Education grant N N518 407438.

We are thankful to Dr. B. Tomanek from National Research Council Canada, Institute for Biodiagnostics (West), Calgary, Canada, and to Andrew Procca from National Research Council of Canada, Institute for Biodiagnostics, Winnipeg, Manitoba, Canada, for critical reading of the manuscript.

Open Access This article is distributed under the terms of the Creative Commons Attribution Noncommercial License which permits any noncommercial use, distribution, and reproduction in any medium, provided the original author(s) and source are credited.

\section{REFERENCES}

1. Li CL, Martini LG, Ford JL, Roberts M. The use of hypromellose in oral drug delivery. J Pharm Pharmacol. 2005;57(5):533-46.

2. Grassi M, Grassi G. Mathematical modelling and controlled drug delivery: matrix systems. Curr Drug Deliv. 2005;2:97-116.

3. Siepmann J, Siepmann F. Mathematical modeling of drug delivery. Int J Pharm. 2008;364:328-43.

4. Sawada T, Sako K, Fukuji M, Yokohama S, Hayashi M. A new index, the core erosion ratio, of compression-coated timed-release tablets predicts the bioavailability of acetaminophen. Int J Pharm. 2003;265:55-63.

5. Dorożyński P, Kulinowski P, Mendyk A, Młynarczyk A, Jachowicz R. Novel application of MRI technique combined with flowthrough cell dissolution apparatus as supportive discriminatory test for evaluation of controlled release formulations. AAPS PharmSciTech. 2010;11(2):588-97.

6. Borgquist P, Körner A, Piculell L, Larsson A, Axelsson A. A model for the drug release from a polymer matrix tablet-effects of swelling and dissolution. J Control Release. 2006;113:216-25.

7. Kiil S, Dam-Johansen K. Controlled drug delivery from swellable hydroxypropylmethylcellulose matrices: model-based analysis of observed radial front movements. J Control Release. 2003;90:1-21.

8. Ju RTC, Nixon PR, Patel MV, Tong DM. Drug release from hydrophilic matrices. 2. A mathematical model based on the polymer disentanglement concentration and the diffusion layer. $\mathrm{J}$ Pharm Sci. 1995;84:1464-77.

9. Colombo P, Bettini R, Santi P, de Ascentiis A, Peppas PN. Analysis of the swelling and release mechanisms from drug delivery systems with emphasis on drug solubility and water transport. J Control Release. 1996;70:383-91.
10. Gao P, Meury RH. Swelling of hydroxypropyl methylcelulose matrix tablets. 1. Characterization ofj swelling using a novel optical imaging method. J Pharm Sci. 1996;85:725-31.

11. van der Weerd J, Kazarian SG. Combined approach of FTIR imaging and conventional dissolution tests to drug release. J Control Release. 2005;98:295-305.

12. Kazarian SG, van der Weerd J. Simultaneous FTIR spectroscopic imaging and visible photography to monitor tablet dissolution and drug release. Pharm Res. 2008;25:853-60.

13. Ferrero C, Massuelle D, Doelker E. Towards elucidation of the drug release mechanism from compressed hydrophilic matrices made of cellulose ethers. II. Evaluation of a possible swellingcontrolled drug release mechanism using dimensionless analysis. J Control Release. 2010;141:223-33.

14. Blumich B. NMR imaging of materials. Oxford: Clarendon; 2000.

15. Richardson JC, Bowtell RW, Mäder K, Melia CD. Pharmaceutical applications of magnetic resonance imaging (MRI). Adv Drug Deliv Rev. 2005;57(8):1191-209.

16. Weglarz WP, Hemelaar M, van der Linden K, Franciosi N, van Dalen G, Windt $\mathrm{C}$, et al. Real-time mapping of moisture migration in cereal based food systems with Aw contrast by means of MRI. Food Chem. 2008;106:1366-74.

17. Rajabi-Siahboomi AR, Bowtell RW, Mansfield P, Henderson A, Davies MC, Melia CD. Structure and behavior in hydrophilic matrix sustained release dosage Forms. 2. NMR-Imaging studies of dimensional changes in the gel layer and core of HPMC tablets undergoing hydration. J Control Release. 1994;31:1218.

18. Kojima M, Ando S, Kataoka K, Hirota T, Aoyagi K, Nakagami $\mathrm{H}$. Magnetic resonance imaging (MRI) study of swelling and water mobility in micronized low-substituted hydroxypropylcellulose matrix tablets. Chem Pharm Bull. 1998;46:324-8.

19. Kojima M, Nakagami H. Investigation of water mobility and diffusivity in hydrating micronized low-substituted hydroxypropyl cellulose, hydroxypropylmethyl cellulose, and hydroxypropyl cellulose matrix tablets by Magnetic Resonance Imaging (MRI). Chem Pharm Bull. 2002;50(12):1621-4.

20. Tritt-Goc J, Piślewski N. Magnetic resonance imaging study of the swelling kinetics of hydroxypropylmethylcellulose (HPMC) in water. J Control Release. 2002;80(1-3):79-86.

21. Kowalczuk J, Tritt-Goc J, Piślewski N. The swelling properties of hydroxypropyl methyl cellulose loaded with tetracycline hydrochloride. Solid State Nucl Magn Reson. 2004;25:35-41.

22. Baumgartner S, Lahajnar G, Sepe A, Kristl J. Quantitative evaluation of polymer concentration profile during swelling of hydrophilic matrix tablets using H-1 NMR and MRI methods. Eur J Pharm Biopharm. 2005;59(2):299-306.

23. Chen YY, Hughes LP, Gladden LF, Mantle MD. Quantitative ultra-fast MRI of HPMC swelling and dissolution. J Pharm Sci. 2010;99:3462-72.

24. Fyfe CA, Grondey H, Blazek-Welsh AI, Chopra SK, Fahie BJ. NMR imaging investigations of drug delivery devices using a flowthrough USP dissolution apparatus. J Control Release. 2000;654 (68):73-83.

25. Dorożyński P, Kulinowski P, Jachowicz R, Jasiński A. Development of the System for Simultaneous Dissolution Studies and Magnetic Resonance Imaging of Water Transport in Hydrodynamically Balanced Systems - A Technical Note. AAPS PharmSciTech. 2007;8: Article 15.

26. Kulinowski P, Dorożyński P, Jachowicz R, Weglarz W. Integrated system for dissolution studies and Magnetic Resonance Imaging of the Hydrodynamically Balanced Systems - a tool for quantitative assessment of hydrogel formation process. J Pharm Biomed Anal. 2008;48:685-93.

27. Nott K. Magnetic resonance imaging of tablet dissolution. Eur J Pharm Biopharm. 2010;74:78-83. 
28. Kulinowski P, Dorożyński P, Jachowicz R, Jasiński A. MRI analysis of moving fronts in floating dosage forms. Acta Phys Pol A. 2005;108:155.

29. Tajarobi F, Abrahmsen-Alami S, Carlsson AS, Larsson A. Simultaneous probing of swelling, erosion and dissolution by NMR-microimaging - effect of solubility of additives on HPMC matrix tablets. Eur J Pharm Sci. 2009;37:89-97.

30. Zeitler JA, Gladden LF. In vitro tomography and non-destructive imaging at depth of pharmaceutical solid dosage forms. Eur J Pharm Biopharm. 2008;71:2-22.

31. Gonzalez RC, Woods RE. Digital image processing. Upper Saddle River: Prentice Hall; 2008.
32. Young IT, Gerbrands JJ, van Vliet LJ. Fundamentals of Image Processing. Delft University of Technology.; 1995-2007. Available from: ftp://ftp.tudelft.nl/pub/DIPimage/docs/FIP2.3.pdf.

33. Kaynig V, Arganda-Carreras I, Cardona A. Trainable Segmentation Plugin. 2010. Available from: http://pacific.mpi-cbg.de/wiki/index. php/Trainable_Segmentation.

34. Chen M-L, Shah VP, Ganes D, Midha KK, Caro J, Nambiar P, et al. Challenges and opportunities in establishing scientific and regulatory standards for assuring therapeutic equivalence of modified release products: workshop summary report. Eur J Pharm Sci. 2010;40:148-53. 